\title{
AUDITORÍA A LOS PROCESOS EN LAS EMPRESAS
}

Mg. Adrián Alejandro Flores Konja*

\section{PRESENTACIÓN}

Las Auditorias para ayudar a sus organizaciones y brindar servicios eficientes, eficaces, económicos y oportunos, precisan comprender la visión estratégica de la Alta Gerencia y la razón por la cual los procesos de la empresa vienen siendo objeto de creciente atención.

En este sentido, se hace necesario poner énfasis en la auditoria a los procesos principales de la empresa e instituciones, pero con un enfoque diferente. Se requiere observarlos en su relación con el ambiente externo a la empresa (mercado, competencia, clientes, cambios tecnológicos) y el ambiente interno (valores, misión, objetivos, estrategias).

A continuación se intenta esbozar de manera resumida y esquemática un marco conceptual que permita entender el contexto básico en que se desarrolla la Alta Gerencia teniendo como punto de referencia permanente la unidad esencial de los negocios: Los Procesos.

En la primera sección se trata algunos conceptos (globalización, mercado, competitividad) que permiten una visión condensada del ambiente externo en que operan las organizaciones. Dichos conceptos nos servirán de constante referencia para llegar al enfoque central: La Auditoría a los Procesos de la empresa.

En la segunda parte de la misma sección se aborda el ambiente interno de la empresa, destacando aquellos factores que deben tenerse en cuenta para asegurar una adecuada gestión gerencial (la estrategia, la estructura y la cultura) exponiéndolos desde el punto de vista de su interacción dinámica sostenida en una base común: Los Procesos de la empresa.

En la segunda sección se centra en los procesos de la empresa, definiéndolos e identificando sus características más importantes y relevando el enfoque a los procesos o visión horizontal del negocio sobre el enfoque funcional o visión vertical.

En la tercera sección exponemos algunos criterios básicos a tener en cuenta para el planeamiento estratégico de auditoría que incorpore la revisión, evaluación y control de los procesos de la empresa por ser actividades claves para el éxito de los negocios, concluimos esta sección recordando brevemente la importancia de algunos parámetros de medición de la gestión gerencial.

* Profesor Principal de la Facultad de Ciencias Contables. 
A continuación en la cuarta sección se presentan algunos lineamientos básicos para el replanteo estratégico, incluye la misión así como los objetivos y metas previstas para la auditoría; todo esto ligado a estrategias minimas que aseguren su cumplimiento y a una estructura y cultura de apoyo donde el papel de la Alta Gerencia es de vital importancia para el logro de los objetivos. Igualmente, se adjunta los diagramas de los procesos considerados con sus unidades de ejecución y sus principales actividades que permitan, posteriormente, la elaboración de programas de Auditoría específicos.

Finalmente, a manera de conclusión señalamos la importancia estratégica que tiene para la Auditoria Interna y la Alta Gerencia, la evaluación y control permanente de los principales procesos de la empresa.

\section{EL CONTEXTO ACTUAL}

\section{El Ambiente Externo}

\section{a. La globalización:}

El mundo contemporáneo viene transformándose necesariamente. Los cambios que experimentan los sistemas políticos, económicos $\mathrm{y}$ sociales en el mundo desarrollado, repercuten en los países de menor desarrollo. La desintegración del bloque socialista marcha paralela con la configuración de un nuevo orden Internacional. De una hegemonía mundial bipolar -representada por Estados Unidos y la URSS- se pasa a una hegemonía económica tripolar, liderada por los Estados Unidos, Alemania y Japón.
Se avanza a la universalización de valores jurídicos, morales, políticos (medio ambiente, derechos humanos, democracia, etc.); los problemas de pobreza, empleo, población, son temas de preocupación mundial. La incesante innovación tecnológica y el desarrollo de las comunicaciones, favorecen la integración de la economia mundial fuertemente influenciada por el modelo liberal de occidente. Los mercados, las inversiones $y$ las finanzas, se expanden y se ven afectadas por las fluctuaciones económicas, financieras, monetarias, etc.

Todos estos procesos, vistos desde la óptica de su internacionalización, interrelación e interdependencia se conceptúan como globalización.

En este escenario, la visión de globalización de la actividad económica incorpora características de complejidad, inestabilidad e incertidumbre.

\section{b. El mercado actual}

En el contexto de globalización de la actividad económica desaparecen las fronteras nacionales entre los mercados. Emerge un mercado mundial abierto sujeto a la ley de la oferta y la demanda.

A la vez el mercado adquiere características de turbulencia. Se vuelve vulnerable a perturbaciones debido al alto índice de interconexiones entre los elementos que componen el entorno. La innovación tecnológica incide en los cambios de los sistemas de producción, comercialización, distribución, etc. 
En esta situación la competencia adquiere vital importancia para las empresas, si no logran el incremento permanente de la productividad y calidad desaparecerán o no progresarán.

\section{c. Las presiones competitivas}

La globalización de la actividad económica favorecida por la apertura de los mercados nacionales, y la expansión de las inversiones transforma el mercado y le incorpora algunas características de este proceso mundial. Así, el entorno en el cual se desenvuelven las organizaciones empresariales es global y no local, turbulento y no estable, agresivo y no amigable.

Del mercado con tales caracteristicas se desprenden tres fuerzas que directa o indirectamente presionan a los negocios a cambiar, denominadas las tres $" \mathrm{C} *$, clientes, competencia, cambio.

\section{El cliente}

Los mercados masivos han desaparecido, actualmente existe el cliente individualizado, exigente, mejor informado sobre las diversas opciones que se le presentan, sabe lo que quiere y como lo quiere. Está consciente de que se constituye en la fuerza dominante en la relación vendedor-cliente; busca los productos y servicio que atiendan sus preferencia y necesidades con calidad, bajo costo y oportunidad.

\section{La competencia}

Con el levantamiento de las barreras comerciales, los mercados nacionales han cambiado.
Ingresa la competencia extranjera con mayor rendimiento y eleva el marco competitivo.

Con el avance de la tecnología, cambia la naturaleza de la competencia surgiendo técnicas de servicio enteramente nuevas. En este ambiente, la competencia se vuelve agresiva, siempre dispuesta a ganar nuevos mercados.

\section{El cambio}

La naturaleza del cambio es distinta a la de épocas anteriores. Hoy en día el cambio es acelerado y permanente; se ve favorecido por el incesante desarrollo tecnológico que contribuye a que se creen nuevos productos y se ofrezcan nuevos servicios.

Las organizaciones empresariales deben esforzarse en detectar la dirección del cambio, desarrollar suficiente poder para moldearlo $\mathrm{y}$ actuar rápidamente para darle respuesta adecuada.

\section{d. La competitividad}

Una empresa es competitiva cuando en función a sus características internas, dentro de un determinado contexto, logra capacidad estructural para generar beneficios. Pero no es suficiente que pueda generar beneficios. Se requiere que éstos sean sostenibles en el tiempo; esto sólo es posible adecuándose constantemente al entorno.

Para lograr ser competitivas las organizaciones buscan estrategias de desarrollo. Éstas pueden enfocarse desde el punto de vista de los logros que esperan alcanzar. 


\section{Las ventajas competitivas}

Una empresa para generar beneficios sostenibles en el tiempo necesita lograr una ventaja competitiva sobre sus competidores.

Se define una ventaja competitiva como cualquier característica de la empresa que la aisla de la competencia directa dentro de un sector, y es sostenible en el mediano y largo plazo.

Las posibilidades para alcanzar ventajas competitivas sostenibles pueden darse a través de:

1. La diferenciación consiste en que el producto o servicio ofrecido por la empresa es, de algún modo, único para sus clientes.

2. El liderazgo en costos: la empresa busca ser capaz de obtener costos globales inferiores a los de sus competidores.

En ambas opciones, el concepto de la cadena de valor permite descomponer la empresa en sus actividades más importantes para analizar cada una de ellas por separado y determinar en cual se pueden desarrollar ventajas competitivas.

\section{La satisfacción del cliente}

Para aumentar su nivel de competitividad en la relación con el cliente, las empresas tratan de satisfacerlo proporcionándole un producto o servicio de calidad ajustado a sus deseos o necesidades.

La calidad se constituye en la respuesta más adecuada a las exigencias del cliente de hoy. Es el resultado, a su vez, de la calidad de la organización, del trabajo, del servicio, del proceso, de las personas, etc.

La calidad total como estrategia busca tanto la calidad del servicio (satisfacción del cliente) como la calidad en el uso de los recursos (productividad del cliente), así como la calidad en el uso de los recursos (productividad a más bajo costo).

Incorporada a los procesos de la empresa, busca el mejoramiento continuo de la calidad de todo el sistema, y el mejoramiento continuo es el soporte para mantener las ventajas competitivas logradas.

\section{La innovación}

Los cambios que se dan en el entorno de los negocios como consecuencia del rápido avance tecnológico, el aumento de los niveles de competencia y el desarrollo de nuevas formas de gestión empresarial, impulsan a las empresas a ver en la innovación un recurso para mantener su competitividad.

Como estrategia, el planeamiento del cambio continuo para mejorar la productividad de los procesos existentes tiene su expresión en la innovación empresarial y en la acción de concebir algo diferente. Es otra manera de hacer las cosas con el objeto de mantener o aumentar la competitividad de la empresa y tener éxito.

Las nuevas ideas y las nuevas formas de hacer las cosas crean ventajas competitivas porque permiten aventajar a los competidores, con nuevos o mejores productos o servicios. Creada una nueva ventaja 
competitiva, ésta sólo se sostiene mediante el mejoramiento, la innovación y el cambio.

\section{El Ambiente Externo}

\section{a. El planeamiento estratégico}

La globalización de los negocios, la desaparición de fronteras entre los mercados, los incesantes cambios tecnológicos y las expectativas de los clientes impactan en las organizaciones empresariales forzándolas a cambiar para lograr mayores niveles de competitividad y poder subsistir.

En esta coyuntura, no es el cambio sino cómo cambiar la principal preocupación de los negocios. De hecho, la forma como se ha venido planeando, organizando, dirigiendo y controlando los negocios ya no responde a la nueva realidad.

Esta situación conduce a las organizaciones empresariales hacia los aspectos realmente estratégicos para sus intereses. Por consiguiente, el actual modelo gerencial enfatiza el planeamiento estratégico empresarial como el factor clave de éxito para conducir éxitosamente a las organizaciones en un ambiente altamente competitivo.

\section{b. Las dimensiones básicas de la empresa moderna}

Bajo las condiciones las cuales se desenvuelve actualmente la gestión empresarial, la dirección para el cambio se enfoca a partir de la relación entre la forma o camino que las empresas siguen en su crecimiento (estrategia), el diseño de la organización (estructura) y el compromiso o grado de participación de su gente (cultura).

\section{La estrategia}

El entorno competitivo donde se desenvuelve la organización requiere un cambio de actitud en la gestión gerencial.

En las condiciones actuales los enfoques gerenciales se orientan a comprender las características presentes y rutinas de la empresa a nivel estratégico, e identificar y considerar qué factores determinarán su éxito en los próximos años.

La estrategia definida como "la determinación de metas y objetivos básicos de largo plazo de la empresa, la adopción de cursos de acción y la asignación de recursos necesarios para lograr dichas metas se constituyen en una guía de acción para la gerencia.

La visión estratégica de la empresa se conceptúa como el enfoque gerencial que trata de lograr un marco de referencia flexible e inspirador para enfrentar un mundo inestable, complejo e incierto. Dicha visión estratégica comprende los siguientes elementos:

a. Los valores se constituyen en guias que sustentan el comportamiento de los miembros de la empresa; pueden referirse a aspectos tales como la importancia de los seres humanos que la integran, el rol de la calidad, el servicio, etc.

b. La misión: partiendo de la descripción de lo que somos ahora define lo que aspiramos "ser" y "hacen". La declaración de la 
misión debe ser comprendida por los miembros de la organización.

Los objetivos: son aquellos logros de largo plazo adoptados entre los múltiples existentes para cumplir con la misión. Las metas son los propósitos especificos y calificativos de corto y mediano plazo encaminados hacia los objetivos.

\section{La estructura}

En función a las estrategias diseñadas se adoptan las medidas para adecuar la estructura organizacional alineando sus roles, sistemas y habilidades como elementos componentes de la misma.

Roles: la organización de las empresas era vertical y funcional (Finanzas, Logistica, Operaciones, Recursos Humanos, Ventas, Servicios, etc), su estructura era rígida, jerarquizada y burocrática. Esta visión vertical se plasmaba en el organigrama tradicional que no reflejaba la interconexión de las unidades, ni la naturaleza de los procesos que éstas realizaban.

Este modelo conduce a que cada área funcional sea responsable de un eslabón pero ninguna de la cadena total. De esta manera se creaba inoperancia y lentitud en los sistemas; los procesos se tornaban complejos poco flexibles, con crecientes niveles de revisión y aprobación.

Al buscar cada área para optimizar aisladamente sus propias actividades, las ganancias en productividad no estaban en relación a la inversión en automatización.
La organización moderna, por el contrario, requiere cambios para que su estructura logre flexibilidad, eficiencia, productividad y calidad. Para estos fines, la estructura que se busca es simple:

- Menos rigidas, preparada para los cambios rápidos.

- Menos niveles, más plana con poco staff.

- Menos funciones, más automatización.

Sistemas: se caracterizan por que son multifuncionales, no se diseñan para funciones en particular. Se orientan a las decisiones estratégicas, y buscan por ejemplo que el flujo de materiales esté previamente coordinado para llegar "justo a tiempo".

En este esfuerzo, los procesos se diseñan orientados a la obtención de mayor productividad en la relación recursos-metas, incorporando estructuralmente la calidad total y orientada todas las actividades hacia el cliente.

Los procedimientos que se implanten deben ser simples, flexibles y preparados para obtener los resultados previstos con la menor cantidad de tareas aplicando el principio de calidad total (disminución de costos, tiempo y errores para un mejor resultado).

Habilidades: la gerencia, al encarar un contexto en continuo cambio y altamente competitivo, requiere cambiar todo el esquema mental con que trabajaba tradicionalmente. Debe acostumbrarse a proyectarse mental y simultáneamente varios escenarios posibles, ajustando a ellos sus decisiones. 
Las condiciones de competitividad impulsan a la gerencia a desarrollar capacidades orientadas al mercado buscando siempre mejoras en la calidad de los productos y servicio que se ofrezcan.

Su visión debe estar orientada a los resultados que quiere, espera o vislumbra.

La innovación es otro elemento importante para el éxito permanente de los negocios; la gerencia moderna debe estimular el cambio y la generación de nuevas ideas y nuevos procedimientos. El pensamiento innovador debe formar parte de la estrategia que direccione los negocios.

\section{- La cultura}

En los tiempos actuales la cultura organizacional juega un papel importante en la gestión exitosa. Definida como la manera tradicional de llevar a cabo las responsabilidades comprende, además, el conjunto de valores, actitudes y compromisos que existen en el ambiente donde trabajan las personas.

Como se mencionó anteriormente, los valores guian y sustentan el comportamiento de los miembros de la empresa. Ciertas definiciones como el menor costo, la mayor eficiencia, la mayor calidad o el mejor servicio al cliente, conducen a las empresas a ser más competitivas.

La cultura tiene marcada influencia en las actitudes de los individuos dentro de la empresa, tanto a nivel individual como grupal; se manifiesta en aspectos como la toma de decisiones, las interacciones personales, las recompensas y sanciones, etc.
También contribuye a que el personal esté comprometido $\mathrm{e}$ involucrado con los objetivos, las metas y las estrategias de la organización.

\section{c. La reingenieria para los cambios}

Para lograr los cambios requeridos a nivel de estrategia, estructura y cultura y alcanzar los objetivos propuestos "la reingeniería de los negocios", expresión del esfuerzo de transformación de toda la organización, y la "reingeniería de los procesos", aplicada solamente al nivel operativo de la empresa, constituyen las herramientas más eficaces para lograr dichos objetivos.

La reingeniería, definida como "la reconcepción de los fundamentos y el rediseño de los procesos de negocios para lograr mejoras sustanciales en las medidas criticas de rendimiento (costos, calidad, servicio y velocidad]", tiene como punto de partida los procesos de la empresa.

\section{LOS PROCESOS EN LA EMPRESA}

\section{a. Concepto de proceso}

Proceso es cualquier actividad o grupo de actividades que emplea un insumo, le agrega valor y obtiene un producto para suministrarlo al cliente externo o interno.

\section{b. Caracteristicas de los procesos}

Emplean insumos

Son el input de los procesos. Considera el material que será 
utilizado para elaborar el resultado final de los procesos.

Agregar un valor

Durante la ejecución de un proceso, se efectúa una transformación o cambio de los insumos empleados obteniendo un producto o servicio que se destinará al cliente.

Utilizan los recursos de la organización

En la ejecución de los procesos se dispone de los recursos humanos, materiales y financieros (medios, personas, equipos, tiempo, capital e inventario).

Tienen un costo

El de los insumos y recursos de la organización involucrados en el proceso.

Producen un resultado

El objetivo de un proceso es lograr un producto o servicio.

Tienen un cliente externo o interno.

Que es el destinatario de lo generado por el proceso.

En su ejecución participan generalmente varias áreas de la empresa.

Mientras la empresa está organizada verticalmente (áreas funcionales) el proceso tiene un flujo horizontal que puede involucrar a varias áreas funcionales. Esta situación podría generar vacios $y$ yuxtaposiciones produciendo un impacto negativo sobre la eficiencia y la efectividad del proceso.
Tiene reglas o procedimientos para su aplicación.

Para ejecutar un proceso se siguen ciertos pasos (formal o informalmente).

\section{c. Importancia del enfoque de los procesos}

Todas las actividades de la empresa constituyen procesos o subprocesos que tienen un costo.

Tradicionalmente, todo el apoyo para la optimización ha estado orientado hacia los procesos de producción. Sin embargo, estudios realizados en Estados Unidos de Norteamérica demuestran que los costos de producción representan únicamente el $10 \%$ del costo del producto; el más significativo es el de los procesos de la empresa que en algunas áreas de servicio alcanzan al $100 \%$ del costo.

La interacción de los clientes es muy importante. No obstante la buena calidad de un producto o servicio, se pueden perder clientes si existen deficiencias en otros procesos de la empresa, que impliquen mayor costo o una mala percepción de la misma.

Los clientes son susceptibles a alejarse debido a procesos mediocres y productos deficientes.

Si antes el enfoque estaba centrado en la organización, ahora debe ser reexaminado y orientado al proceso.

En el siguiente cuadro, mostramos las diferencias entre ambos enfoques: 


\begin{tabular}{|c|c|}
\hline CENTRADO EN LA ORGANIZACIÓN & $\begin{array}{l}\text { CENTRADO EN EL } \\
\text { PROCESO }\end{array}$ \\
\hline - Los empleados son el problema & - El proceso es el problema \\
\hline - Empleados & - Personal \\
\hline - Hacer un trabajo & - Ayudar a que se haga el trabajo \\
\hline - Comprender el trabajo & $\begin{array}{l}\text { - Saber que lugar ocupa el trabajo en el } \\
\text { proceso }\end{array}$ \\
\hline - Evaluar a los individuos & - Evaluar el proceso \\
\hline - Cambiar a la persona & - Cambiar el proceso \\
\hline $\begin{array}{l}\text { - Siempre se puede encontrar un empleado } \\
\text { mejor }\end{array}$ & - Siempre se puede mejorar el proceso \\
\hline - Motivar a las personas & - Eliminar barreras \\
\hline - Controlar a los empleados & - Desarrollo de las personas \\
\hline - No confiar en nadie & - Trabajar en equipo \\
\hline - ¿Quién cometió el error? & - ¿Qué ocasionó el error? \\
\hline - Corregir errores & - Prevenir errores \\
\hline $\begin{array}{l}\text { - Orientado al producto o servicio más } \\
\text { importante }\end{array}$ & - Orientado al cliente \\
\hline
\end{tabular}

\section{AUDITORÍA A LOS PROCESOS}

\section{a. El planeamiento estratégico de Auditoria y el enfoque a los procesos.}

La auditoria, consciente de su compromiso con la organización, de ofrecer servicios eficientes, eficaces, económicos $\mathrm{y}$ oportunos, debe orientarse al planeamiento estratégico de auditoria con la visión del control permanente de la ejecución de las estrategias empresariales.

Pasando de una auditoria funcional a desarrollar una auditoría orientada a los procesos (flujos de actividad, información, decisión y recurso).

Este proceso comprende la definición de los siguientes elementos.
Misión: tomando como base lo que soinos ahora establece lo que aspiramos ser.

Factores críticos de éxito: nos permite identificar aquellos elementos necesarios para alcanzar la misión que hemos definido. Por ejemplo, pueden ser considerados factores críticos de éxito la imagen profesional que proyectan individual y grupalmente los auditores, la calidad del personal, etc.

Fuerza y debilidades: se debe tener comprensión de nuestras fuerzas y debilidades en relación a las expectativas de la gerencia y a las necesidades percibidas para alcanzar los factores críticos de éxito. 
Oportunidades y amenazas: éstas provienen para este caso, fundamentalmente de las innovaciones tecnológicas debiendo capitalizarlas o evitarlas según sea el caso.

Objetivos y metas: son aquellos logros a largo plazo hacia los cuales se dirigen los esfuerzos para que la misión no quede en el ámbito de las intenciones.

Estrategias: define como se va a lograr los objetivos básicos de la auditoría.

Plan de acción: en este aspecto culmina formalmente el planeamiento estratégico. Se fijan las acciones necesarias para aplicar las estrategias y conjuntamente con ellas se definen las responsabilidades, los recursos necesarios, los plazos y logros esperados.

\section{b. Utilización de indicadores de gestión}

Los indicadores de gestión tienen importancia dentro de las técnicas de Auditoría.

La combinación de objetivos bien planeados, una organización firme y dirección capaz, así como la motivación, tendrán pocas probabilidades de éxito si no existe un sistema adecuado de control que permita determinar desviaciones respecto al plan estratégico y visualizar si el desarrollo de las operaciones está contribuyendo al logro de la misión, los objetivos y las estrategias empresariales.

Los indicadores de gestión deben cumplir las siguientes características claves: ser simples, estar estrechamente relacionados a los procesos y tener gran flexibilidad para adecuarse a las estrategias gerenciales.

De esta perspectiva se pueden definir tres categorias de indicadores de gestión:

Efectividad: cuantifica la bondad de un producto o servicio y debe ser medida en función al grado hasta el cual los OUTPUTS del proceso o subprocesos satisfacen las necesidades y expectativas de calidad de los clientes internos y externos. Efectividad es tener el OUTPUTS apropiados en el lugar apropiado, en el momento apropiado y para los clientes externos al precio apropiado.

Eficiencia: mide en términos económicos los resultados de la organización en su conjunto, así como de los productos o servicios que ofrece y de sus distintos procesos.

Se alcanza cuando los recursos se minimizan y se elimina el desperdicio en búsqueda de efectividad. A través de la productividad es posible tener una medida de la eficiencia.

- Adaptabilidad: cuantifica la eficiencia de un proceso y la velocidad de respuesta de toda la organización a las expectativas futuras y cambiantes del cliente y los requerimientos especiales e individuales del cliente de hoy.

\section{c. Establecimiento de medidas de control}

Se dice "si usted no puede medir lo que hace, no puede controlarlo. Si no puede controlarlo, no puede 
dirigirlo. Si no puede dirigirlo, no puede mejorarlo". Esta expresión, describe con realismo la importancia de contar con medidas que permitan evaluar la gestión gerencial.

Un punto de referencia en el proceso de evaluación de la eficiencia y efectividad con que se están conduciendo los procesos de la empresa constituyen las medidas y objetivos establecidos para los procesos. Éstos deben haber sido definidos por la gerencia y nos permiten entender los resultados que se han propuesto lograr.

Desde el punto de vista del auditor, en el establecimiento de medidas de control gerencial, es importante considerar:

En las medidas de efectividad: para que el proceso sea efectivo, deben haberse definido las necesidades y expectativas del cliente a fin de determinar si esas necesidades están siendo satisfechas. Estas necesidades y expectativas deben haber sido descritas específicamente en términos mensurables.

Por lo general las necesidades y expectativas de los clientes internos $y$ externos se relacionan con:

Exactitud

Puntualidad

Rendimiento

Responsabilidad

Confiabilidad

Costos

Uso fácil

Rapidez

Atención
En las medidas de eficiencia: aunque la primera es satisfacer las necesidades y expectativas del cliente, la eficiencia debe lograrse primero. A medida que se reducen los costos operacionales parte de los ahorros deben trasladarse al cliente externo.

Cada proceso o subproceso debe tener un minimo de dos requerimientos de eficiencia establecidos:

Uno que constituya una relación beneficio/costo o recurso por unidad de OUTPUT (por ejemplo costo por solicitud de compra procesada, costo por nuevo empleado) y otro que mida el tiempo del ciclo (horas para procesar una orden de compra, dias para contratar un empleado).

Son medidas tipicas de eficiencia.

Tiempo de procesamiento

Recursos contados por unidad de output

Costo del valor agregado por unidad de output

Costo de mala calidad

Tiempo de espera por unidad.

En las medidas de adaptabilidad: la calidad comprende la satisfacción de los requerimientos del cliente; sin embargo, para muchos clientes de hoy lo suficientemente bueno ya ha dejado de serlo. Los procesos deben tener la capacidad de ajustarse no solo para satisfacer las expectativas del cliente promedio, sino también para suministrar información a los procesos de manera que éstos puedan satisfacer las necesidades $\mathrm{y}$ expectativas futuras del cliente. Las posibles medidas en este aspecto son: 
Tiempo promedio para procesar la solicitud especial de un cliente en comparación con los procedimiento estándares;

Porcentaje de solicitudes especiales devueltas.

\section{d.Identificación de los procesos criticos}

El planeamiento de la auditoría debe obtener suficiente información sobre todo los procesos de la empresa identificando los principales y que resultan críticos para el logro de sus objetivos estratégicos empresariales.

Por lo general dentro de la organización coexisten dos tipos de proceso. Uno de ellos está organizado a lo largo de las líneas funcionales, recibe su material de un solo departamento y genera su producción dentro de éste. Estos procesos simples normalmente son subprocesos de proceso, mucho más complejos denominados procesos interfuncionales que fluyen horizontalmente a través de varias funciones o departamentos.

Por regla general ninguna persona tiene individualmente la responsabilidad final de todos los procesos que pueden ser:

Desarrollo de nuevos servicios o productos

Informática

Abastecimiento

Contratación

Facturación y cobro

Servicio o producto de post venta Capacitación.
Cada uno de los procesos puede descomponerse en sus correspondientes subprocesos.

\section{e. Selección de procesos}

Es importante establecer criterios para la selección de los procesos a ser auditados. Puede desperdiciarse gran cantidad de esfuerzo si se seleccionan los procesos equivocados.

Razones para seleccionar un proceso:

Alto impacto en los ingresos de la empresa

Procesos de alto costo

Procesos con tiempo de ciclo prolongados

Problemas y/o quejas de los clientes externos e internos

Existencia de nuevas tecnologias Antecedentes de dolo o fraude.

Para la selección de un proceso debe tenerse en cuenta los siguientes aspectos:

Impacto en el cliente

Impacto sobre la empresa

Impacto sobre el trabajo

Un método objetivo para establecer prioridades en los procesos a ser auditados en la empresa considera los siguientes principios:

Centrarse en las actividades tanto correctivas como preventivas

Hacer énfasis en las áreas que tengan potencial de mejoramiento Trabajar en un número de procesos que sea manejable. 
Se debe recolectar datos reales de los clientes y de las operaciones internas; esto requiere un mayor tiempo para planificar las actividades. En esta labor se emplean los siguientes medios:

Conocer los requerimientos del cliente externo.

Evaluar la importancia de los procesos de la empresa.

Evaluar las oportunidades de mejoramiento de los procesos a través de las recomendaciones.

Seleccionar los procesos críticos.

\section{f. Diagramas de flujo}

Son herramientas clave para la comprensión del funcionamiento interno y las relaciones entre los procesos de la empresa.

Se define como un método para describir gráficamente un proceso existente o uno nuevo propuesto mediante la utilización de símbolos, lineas y palabras simples, demostrando sus actividades y su secuencia en el Proceso.

Mediante la utilización de esta técnica, se logra:

Revelar y describir cualquier proceso, desde el más simple al más complejo

Mediante la comparación del diagrama de flujo de actividades del proceso real, resaltar las áreas donde los procedimientos confusos interrumpen la calidad y productividad.

Representar gráficamente las actividades que conforman un proceso.

Elaborar un diagrama de flujo para la totalidad del proceso hasta llegar al nivel de tarea es la base para analizar y auditar los procesos. Existen muchos tipos de diagrama de flujo y cada uno tiene su propósito. Los más utilizados son:

1. Diagrama de bloque, que proporciona una visión rápida del proceso.

2. Diagrama de flujo funcional, que muestra el flujo del proceso entre áreas.

\section{CONCLUSIONES}

La globalización es un fenómeno que afecta todos los aspectos de la actividad humana y entre ellos las actividades económicas. Así, con la globalización desaparecen las fronteras nacionales entre los mercados y emerge un mercado mundial abierto, sujeto a la ley de la oferta y la demanda.

Este mercado incorpora caracteristicas de globalidad, de turbulencia, al volverse vulnerable a perturbaciones de diversos origenes (económicos, políticos, financieros, etc.), de competencia agresiva, que surge de la lucha de las empresas para ganar nuevos clientes.

Del mercado con estas características se desprenden tres fuerzas que directa o indirectamente presionan a los negocios a cambiar, y a las que se les ha denominado las tres "C" clientes, competencia y cambio.

Los negocios en este entorno, deben desarrollar estrategias que les permitan mantener y alcanzar mayores niveles de competitividad. Estas estrategias pueden orientarse al logro de la satisfacción del cliente a través de la calidad total como 
herramienta competitiva, a la obtención de ventajas competitivas que la diferencian de la competencia, y al enfoque innovador en la gestión gerencial como recurso para mantener su competitividad.

El actual modelo de gestión gerencial tiene como factor clave de éxito para desenvolverse en un ambiente altamente competitivo al planeamiento estratégico, como proceso básico para definir la misión y los objetivos empresariales a largo plazo, y adecuar sus estrategias, su estructura y su cultura al cumplimiento de estos fines.

La aplicación de los cambios requeridos en los ámbitos de la estrategia, la estructura y la cultura, tienen en última instancia, expresión en todas las actividades de la empresa, y éstas se realizan a través de procesos o subprocesos, es mediante los procesos, como esencia de los negocios, donde empiezan los cambios organizacionales.

\section{En conclusión:}

a. Se ha desarrollado una serie de conceptos que intentan describir el ambiente externo e interno en que operan las organizaciones, para entender el contexto básico en que se desarrolla la Gestión Gerencial y comprender que los factores claves para alcanzar, mantener y elevar la competitividad de las empresa reside en ultima instancia en implementar los cambios y las mejoras necesarias a los procesos.
Teniendo en cuenta lo anterior adquiere especial importancia la auditoría a los procesos de la empresa para responder correctamente a las expectativas y necesidades de la Alta Gerencia.

b. El planeamiento de la auditoría a los procesos debe identificar los procesos que resultan críticos para el logro de los objetivos estratégicos empresariales.

c. En la selección de los procesos a ser auditados debe tomarse en cuenta las siguientes razones:

Alto impacto en los ingresos de la empresa

Procesos de alto costo

Procesos con tiempo de ciclos prolongados

Problemas y/o quejas de los clientes externos y internos

Existencia de nuevas tecnologías

d. En la Auditoría a los procesos, es importante la utilización de diagramas de flujo, ya que éstos permiten resaltar las áreas donde los procedimientos confusos interrumpen la calidad y la productividad.

Los diagramas de flujo más utilizados son:

Diagrama del bloque, que proporciona una visión rápida del proceso.

Diagrama de flujo funcional, que muestra el flujo de proceso entre Áreas. 


\section{BIBLIOGRAFÍA}

1. Skinner y Anderson. Auditoría Analitica. Editorial Anaya, España.

2. Carlos Etcheyenne. Calidad Gerencial. Ediciones Macchi, Buenos Aires.

3. Michael Hammen y James Champy. Reingenieria.

4. Carlos A. Slosse. Auditoría un Nuevo Enfoque Empresarial. Ediciones Macchi, Buenos Aires.
5. (Miller) Larry P. Bailey. Guía de Auditoria, Normas de Auditoría (SAS). Editorial Harcourt Brace, España.

6. Brink UIT. Auditoria Interna Moderna. Evaluación de Operaciones y Controles. Editorial Ecasa, México.

7. Fred R. David.Conceptos de Administración Estratégica. 5. ${ }^{\text {a edic. }}$

8. Alvin A. Arens y James K. Loebbecke. Auditoría un Enfoque Integral. Prentice Hall Hispano Americana S.A.

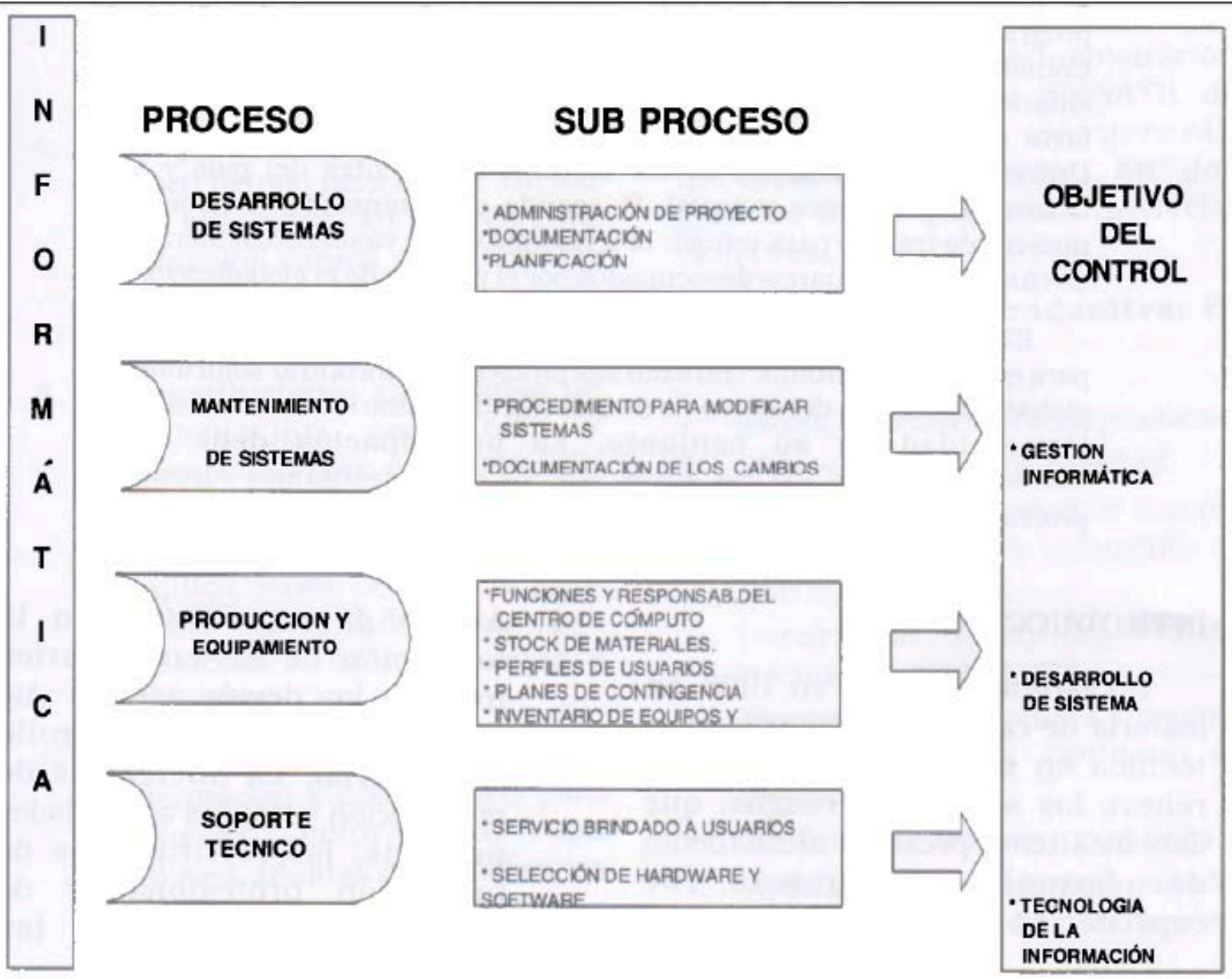

